\title{
Essais
}

ESSAIS

Revue interdisciplinaire d'Humanités

Résister entre les lignes

\section{Octavio Paz et Cornelius Castoriadis : résister à la corruption du langage}

Laurent Leylavergne

\section{(2) OpenEdition}

1 Journals

Édition électronique

URL : http://journals.openedition.org/essais/4552

DOI : $10.4000 /$ essais. 4552

ISSN : 2276-0970

Éditeur

École doctorale Montaigne Humanités

Édition imprimée

Date de publication : 15 juillet 2016

Pagination : 96-109

ISBN : 978-2-9544269-8-3

ISSN : 2417-4211

Référence électronique

Laurent Leylavergne, « Octavio Paz et Cornelius Castoriadis : résister à la corruption du langage », Essais [En ligne], 9 | 2016, mis en ligne le 23 octobre 2020, consulté le 29 octobre 2020. URL : http:// journals.openedition.org/essais/4552 ; DOI : https://doi.org/10.4000/essais.4552 


\title{
Octavio Paz et Cornelius Castoriadis : résister à la corruption du langage
}

\author{
Laurent Leylavergne
}

"Quand une société se corrompt, la première chose qui se gangrène, c'est le langage $»^{1}$ affirmait Octavio Paz. Le phénomène n'est certes pas nouveau ; à la suite de la guerre du Péloponnèse, Thucydide en témoignait déjà à propos de la décadence d'Athènes. Cette corruption, en revanche, n'a sans doute jamais été aussi loin, d'abord et avant tout à travers le phénomène totalitaire, le règne du mensonge et de la mystification, mais aussi, bien que d'une autre façon et à un autre degré, à travers l'univers publicitaire et le règne de la marchandise : corruption et appauvrissement de la signification, destruction de la pensée libre et critique, atteinte des capacités d'écoute, de dialogue et d'actions autonomes. Mais, comme la modernité, la parole au XX' siècle n'est pas d'un seul tenant; elle s'incarne également dans les mots de la résistance, des grandes subversions et de la création autonome - comme le montrent justement les itinéraires et les œuvres d'Octavio Paz et de Cornelius Castoriadis. De la résistance au fascisme et au nazisme à la critique du totalitarisme dit, par antiphrase, "soviétique ", des rencontres communes avec le surréalisme à la critique du conformisme et de l'insignifiance des sociétés contemporaines, l'un et l'autre, à leurs risques et périls, ont pensé et exprimé ces différentes résistances à la dégradation de la parole dans l'espace public. Ils ont également chacun réfléchi, à l'épreuve de ces expériences, ce qui fait le langage et ce que peut signifier la création poétique. En quoi une langue peut-elle être l'objet d'une instrumentalisation corruptrice, réduite à une dimension de code ? Comment penser les effets politiques et psychiques de cette corruption au regard des projets d'émancipation et d'autonomie ? En quoi la parole et l'écriture politiques et poétiques sont-elles susceptibles d'échapper à la ruine du langage et de participer à un renouveau démocratique ? En quoi enfin la dimension poétique du langage demeure comme un besoin fondamental de notre humanité, face aux périls et aux désastres modernes?

1 "Post-scriptum " (1970) au Labyrinthe de la solitude (1950), cité dans Octavio Paz, Solo à deux voix, entretiens avec Julián Ríos, Ramsay, Paris, 1992, p. 27. 
Telles seront les interrogations qui jalonneront ma réflexion sur la ruine du langage, en articulant les itinéraires croisés de Paz et Castoriadis et la manière dont ils incarnèrent une résistance créatrice à ce péril, aux questions plus générales sur le pouvoir des mots et l'élément poétique du langage ${ }^{2}$.

\section{Du goût pour les mots}

Même si le poète mexicain et le philosophe grec ne semblent s'être vraiment connus et liés d'amitié qu'à la fin de leur vie ${ }^{3}$, il est frappant de constater les nombreux liens qui les unissaient, avant même qu'ils se rencontrent vraiment. Parmi ces points de convergence, il y a d'abord une sensibilité originaire à l'imaginaire et une passion pour le savoir : animés tous deux dès l'enfance par une curiosité intellectuelle universelle, un sens de l'étonnement renouvelé devant ce qui est, ils dévorent les livres dans la bibliothèque familiale, se nourrissent de poésie ancienne et moderne (Castoriadis a traduit Rilke en grec, Paz écrit de la poésie dès le lycée en lisant Valéry ou Garcia Lorca), de musique, et ne cesseront jusqu'à leur mort de "voir la réalité de tous les jours avec le regard du premier jour " ${ }^{4}$. Au sujet de leur " amor intellectualis" (Ortega y Gasset), autrement dit de ce qui les poussait à s'intéresser à tous les domaines de la connaissance et des arts, il n'est pas anodin de trouver dans la bouche de deux de leurs contemporains une remarque analogue : Claude Lévi-Strauss voyait en Octavio Paz « le dernier homme de la Renaissance " et Pierre VidalNaquet n'hésitait pas à comparer Castoriadis à Pic de la Mirandole ou Diderot $^{6}$. Ce qui semble avoir frappé les contemporains de ces deux écrivains, c'est ainsi le souffle vivant d'une pensée et d'une parole faisant feu de tout bois et ne renonçant jamais à la tâche de participer à l'émancipation de chacun en " osant savoir " et en osant " changer la façon commune de penser " pour reprendre les mots des hommes des Lumières. Pourraient aussi s'appliquer à

2 Cet article ne prétend en rien proposer une comparaison exhaustive et systématique entre l'œuvre de Castoriadis et celle de Paz, d'autant plus que cette dernière demanderait bien d'autres analyses, seulement esquissées ici.

3 Aux alentours de 60 ans pour Castoriadis, à l'approche des 70 pour Paz, grâce à leur amitié commune avec un autre " exilé " grec, Kostas Papaïoannou. Pour les détails biographiques, je m’appuie sur l'autobiographie intellectuelle de Paz, Itinéraires, Arcades Gallimard, Paris, 1996, et les ouvrages de Christopher Dominguez Michael, Octavio Paz dans son siècle, Gallimard, Paris, 2014, et de François Dosse, Castoriadis, Une vie, La Découverte, Paris, 2014. Personne ne précise exactement quand leur amitié a commencé (on sait que celle entre Paz et Papaïoannou débuta dès 1946). Le moment le plus probable semble être la fin des années 70 , avec notamment l'intérêt porté par Paz à deux essais de Castoriadis parus en 1978 : le premier sur l'imagination et Aristote ("La découverte de l'imagination »), le second, un texte-bilan, "Le régime social de la Russie " (publié ensuite, à l'initiative de Paz, dans la revue mexicaine Vuelta).

4 Itinéraires, op. cit.

5 Cité in Euvres de Paz, Bibliothèque de La Pléiade, Gallimard, Paris p. XII.

6 Hommage à Castoriadis (1998), sur le site www.castoriadis.org/fr. 
tous deux cette remarque de Castoriadis concernant les grands représentants du courant critique de la modernité artistique : « Breton, Ernst, Joyce, Pound ou, aujourd'hui, Octavio Paz sont infiniment plus savants sur notre tradition culturelle et nourris par elle que les peintres pompiers ou les membres de l'Académie française ${ }^{7}$. Avant de revenir sur ce courant de la grande subversion, on peut souligner cet amour commun de la poésie, de sa puissance magique et multiforme, passion qui n'est sans doute pas pour rien dans les réticences instinctives de l'un et l'autre à l'égard de la parole manipulatrice ou plus encore à l'encontre de la lignification du langage opérée par le totalitarisme : « Il suffisait de lire cinq lignes de Staline pour comprendre que la révolution ne pouvait pas être ça ${ }^{8}$. Le sens critique et le gồt pour la beauté des mots comme du monde ne sont pas à comprendre ici comme le résultat d'une formation intellectuelle qui, en elle-même, ne garantit en rien contre l'aveuglement et la bêtise politiques, mais plutôt comme la source même de leurs passions intellectuelles, de leur amour du débat et de leur capacité d'écoute.

Car au fond, leur libido sciendi ne ressort pas d'un enfermement intellectualiste dans la tour immense des lettres et des arts. Il y a chez l'un et l'autre un attachement sensible à la communauté des hommes d'où toute parole émane : le poète et le philosophe puisent à la source vive de ce qui circule entre les hommes « pour communiquer et perpétuer leurs expériences, leurs passions, leurs espérances et leurs croyances " . Même si cet "aliment » demande à être transfiguré et recréé, réélaboré et élucidé, c'est d'abord là que se trouvent l'élément poétique et la réflexion critique : " énorme masse toujours en mouvement, s'engendrant sans cesse, ivre de soi ", telle est bien la langue d'une communauté d'où " jaillissent des images, des jeux de mots, des expressions surgies du néant ", des jugements ingénieux et des questionnements lucides, " sur des lèvres d'enfants, de fous, de savants, d'imbéciles, d'amoureux ou de solitaires "..${ }^{10}$ Ce langage vivant et commun, s'ils l'ont trouvé et recherché, dans le partage des vies ordinaires, dans le Mexico comme l'Athènes d'avant la Seconde Guerre Mondiale, dans Barcelone assiégée comme dans la Grèce en résistance, dans le Paris populaire et cosmopolite de l'immédiat après-guerre, ils ont en même temps assisté à son inexorable dégradation. Un double paradoxe concernant la situation du poète et du philosophe dans la modernité doit être réfléchi, qui nous fera mieux cerner de quelles résistances il est question et de quoi le langage humain moderne est fait. Il y a d'abord

7 Entretien à l'Événement du jeudi, 26 mars-1 ${ }^{\mathrm{er}}$ avril 1992.

8 C. Castoriadis, «Les intellectuels et l'histoire» (1987), in Le monde morcelé, Seuil, Paris, 1990, p. 111.

9 Octavio Paz, Lárc et la lyre (1956), Gallimard, Paris, 1993, p. 45. Le 28 août 1982, Castoriadis écrit à Paz: «En lisant l'Arc et la lyre, j’ai été plus que frappé par la proximité de ce que nous disons l'un et l'autre ; je regrette de ne pas l'avoir lu plus tôt ", in François Dosse, op. cit., p. 461.

10 Ibid., p. 39. 
quelque chose d'inhérent à leur condition même. Fondamentalement, le poète déjoue, selon Paz, les fonctions instrumentales et habituelles de la langue et redonne aux mots leur saveur et "valeurs plastiques, sonores et émotives " ${ }^{11}$ : la première des "résistances ", c'est celle des habitudes linguistiques et de la fonction essentiellement pratique du langage, autrement dit tout ce qu'il y a de figé, d'habituel, d' "inerte " au cœur de nos échanges immédiats, là où le mot "s'amenuise et s'estompe " "en pure monnaie d'échange ». Si le philosophe a quelque chose à voir avec le poète, bien qu'il travaille à élucider et expliquer plutôt qu'à enchanter et révéler par la puissance des mots, c'est bien dans l'effort qu'il doit produire pour surmonter cette pente et s'affronter, comme le poète et autrement que lui, aux " couches les plus profondes de l'être " (Paz), y compris, selon Castoriadis, de ce qui relève du chaos et que le cours de nos vies s'efforce d'atténuer et de voiler ${ }^{12}$.

Mais cette autre résistance que le poète et le philosophe opposent à l'institué et au fonctionnel, elle ne surgit pas dans le néant, elle puise au cour même de ce qui fait le langage, "poésie à l'état naturel » dit Paz : "L'homme est homme grâce au langage, c'est-à-dire grâce à la métaphore originelle qui le rendit autre et le sépara du monde naturel. L'homme est un être qui s'est créé lui-même en créant un langage. Par la parole, l'homme est une métaphore de lui-même. " ${ }^{13}$ Pour le dire autrement, le domaine de l'homme, c'est ici le monde de la représentation et de la signification : il se meut dans un univers de signes, de symboles, de métaphores, bref de tout ce qui est là pour autre chose que lui-même (le "quid pro quo " disait Castoriadis), à partir précisément de "la capacité de voir dans une chose ce qu'elle n'est pas, de la voir autre qu'elle n'est " ${ }^{14}$, faculté aussi bien de poser et de faire surgir des images et des significations (inventer des mots et mettre en relation un signifiant et un signifié, qui n'ont par définition aucun lien naturel) que d'entendre et de voir dans ce qui se dit et se parle autre chose que du bruit informe - c'est ce que Castoriadis a appelé « l'imaginaire radical ». Et ainsi, en bousculant les paroles instituées, le poète et le philosophe réinsufflent dans la langue et la vie commune ce qui est précisément leur source vive.

Le second paradoxe tient à la condition moderne. Dans la vie administrée et morcelée des sociétés de masse, dans "le cauchemar climatisé » du désert marchand, le langage commun se détériore et se pervertit « en jargon desséché de techniciens et de journalistes ", "le langage de la cité tend à se pétrifier en formules et "slogans" et connaît ainsi le même sort que l'art populaire devenu

11 Paz, L'arc et la lyre, op. cit., p. 51 ; les propos qui suivent sont issus du même ouvrage, p. 57 et p. 47.

12 Castoriadis, "L'écrivain et la démocratie " (1988), in Fenêtre sur le chaos, Seuil, Paris, 2007, p. 101.

13 L'arc et la lyre, op. cit., p. 38.

14 L'institution imaginaire de la société, Seuil, Paris, 1975, p. 177. 
produit industriel $»^{15}$. Et, plus encore, lorsque, devant ce vide, monte irrésistiblement la volonté délirante de " reconstruire " un peuple, par la mobilisation de masse et par la haine, que devient le langage sinon l'instrument des pires désastres? Comment résister à ces courants profonds? Comment même parvenir à créer et faire ouvre ? Certains, philosophes ou poètes, et pas des moindres (Heidegger ou Sartre, Aragon ou Neruda) ont trouvé leur place en se faisant ici ou là "fonctionnaires " du monstre bureaucratique totalitaire, " propagandistes » et "idéologues » des " maîtres au pouvoir » ${ }^{16}$.

\section{De la guerre civile}

Réunis par un commun amour de la poésie et du savoir, quels chemins ont dès lors emprunté Castoriadis et Paz en ces sombres temps? Le deuxième point de convergence est à la fois politique et historique et tient à leur engagement commun et à leur expérience de la guerre civile.

Ainsi, en 1937, Octavio Paz, farouche défenseur de la cause républicaine espagnole participe au Congrès International des écrivains antifascistes à Valence où il va rencontrer Pablo Neruda, Louis Aragon et bien d'autres " compagnons de route " venus peu ou prou cautionner l'emprise « soviétique " au nom de l'antifascisme. Malgré son adhésion au communisme (et non au parti), " une réserve tenace, invincible » ne cesse de grandir chez Paz, devant les menaces de "commissaires transformés en théologiens $~^{17}$, et surtout, devant la terrible répression qui s'abat sur les opposants à la mainmise du Parti Communiste au sein du camp républicain : les membres du POUM (proche du trotskisme), les anarchistes de la CNT, exécutés par milliers... Parmi eux, retrouvé par hasard dans les rues de Barcelone quelques jours avant son assassinat, traqué, terrifié, incapable de trouver ses mots, il y eut le Catalan José Bosch Fonserré, ami de combats étudiants au Mexique, éveilleur d'Octavio Paz - il lui avait fait découvrir Kropotkine, Élisée Reclus, Proudhon, Francisco Ferrer. Comme le dit George Orwell, témoin lucide de la tragédie espagnole, le langage politique totalitaire avait rendu « le mensonge crédible » et "le meurtre respectable ${ }^{18}$. Sensible, comme Simone Weil ${ }^{19}$, à la violence qui gangrène tout le camp républicain, troublé face à la montée irrésistible

15 L'arc et la lyre, op. cit., p. 46 et 49.

16 Ibid., p. 47-48.

17 Itinéraires, op. cit., p. 66.

18 "La politique et la langue anglaise " (1946), in Tels, tels étaient nos plaisirs et autres essais, IvréaEncyclo. des Nuisances, Paris, 2005, p. 160. Pour Orwell, à des degrés divers, ces phénomènes sont présents dans tout langage politique...

19 Dans sa Lettre à Georges Bernanos (mai 1938), in Euvres, Quarto - Gallimard, Paris, 1999, p. 405-409; Paz revient sur ce point dans Une planète et quatre ou cinq mondes. Réflexions sur l'histoire contemporaine, Folio Gallimard, Paris, 1985, p. 230. 
de l'emprise "soviétique ", Octavio Paz, de retour au Mexique, s'éloigne du communisme existant et refuse de cautionner les discours de haine contre la "déviance " trotskiste en tombant dans les " méprisables sophismes " des revues qu'il côtoie ${ }^{20}$. Surviennent alors le pacte germano-soviétique en 1939 (“j'ai senti alors que, non seulement on nous avait rogné les ailes, mais qu'on nous avait coupé la langue, que pouvions-nous dire?" ${ }^{21}$ (je souligne), l'assassinat de Trotski à Mexico même en 1940, et enfin la rencontre décisive avec des dissidents européens fuyant les périls totalitaires, en particulier Victor Serge (1890-1947) et Benjamin Péret (1899-1959), surréaliste de la première heure, engagé auprès du POUM et de la colonne Durutti durant la guerre d'Espagne. Parmi tous les exilés, ils tranchent par leur humanité et leur générosité avec la "sécheresse " et la "pédanterie " de leurs compagnons d'infortune : Paz se dit frappé par le fait que «les autres membres du groupe avaient gardé de leurs années marxistes un langage hérissé de formules et de sèches définitions " (je souligne), comme " prisonniers de la scolastique marxiste». Aux contacts de Péret et Serge, s'opère une véritable déprise à l'égard de cette " prison de concepts " dans laquelle s'étaient enfermés, chacun à leur manière, les marxistes orthodoxes comme les dissidents persécutés, à l'image de Trotski auquel Paz appliquait cette formule ${ }^{22}$.

De son côté, Castoriadis, militant très jeune dans le mouvement communiste grec, rejoint, dans la résistance à l'occupant nazi, les rangs " trotskistes " (avec Spiros Stinas). Les quelques illusions sur la possibilité de corriger les "déviations " (notamment chauvines) du PC grec s'effacent devant une expérience fondatrice : "Le caractère réactionnaire du parti communiste, de sa politique, de ses méthodes, de son régime interne, autant que le crétinisme imprégnant, alors comme maintenant, n'importe quel discours ou écrit émanant de la direction $d u P C$, apparaissaient dans une clarté aveuglante $»^{23}$ (je souligne). Toute la verve ironique de Castoriadis s'exprime ici, mais ce jugement tranchant témoigne de la force et de la gravité d'une véritable "révélation " quant au totalitarisme russe, à la mystification du langage stalinien, mais aussi quant à l'inanité des atermoiements trotskistes sur le caractère prétendument "ouvrier " de l'État russe, régime dont la stratégie et la conduite du PC grec révélaient en miroir la nature profonde. Echappant de peu à la mort, Castoriadis prend physiquement conscience, avec les événements de décembre 1944, que la menace pouvait venir aussi bien de la Gestapo, de la droite royaliste que des liquidateurs staliniens du « Guépéou local ». Quittant Athènes avec Kostas Axelos (1924-2010) et Kostas apaïoannou (1925-1981)

20 Itinéraires, op. cit., p. 69.

21 Ibid.

22 Ibid., p. 73.

23 "Introduction" (1972) à La société bureaucratique, Christian Bourgois (éd.), Paris, 1990, p. 21. 
en décembre 1945 sur le Mataroa, ce «fameux bateau " " chargé d'intellectuels d'extrême-gauche qui devaient nourrir et féconder la culture française ${ }^{24}$, Castoriadis continue de militer à Paris, dans un premier temps au sein de la $\mathrm{IV}^{\mathrm{e}}$ Internationale (y rencontrant une première fois Benjamin Péret, sans qu'ils parviennent à s'entendre), puis, faisant éclater cette "prison de concepts » dont parlait Paz, il forge une parole nouvelle, celle qui prendra corps avec Claude Lefort et une poignée de militants dans l'aventure du groupe revue "Socialisme ou Barbarie " (S. ou B. ou S. ou B. pour la revue), fondé en 1949. D'emblée s'énonce, dans une liberté de ton féroce contre toutes les oppressions, à l'Est comme à l'Ouest, une réflexion d'ampleur, inédite et plurielle contre le " capitalisme bureaucratique », totalitaire ou non, contre le grand " mensonge déconcertant " en URSS - " quatre lettres quatre mensonges » aimait à répéter Castoriadis après Souvarine. Malgré la rupture radicale à l'égard du marxisme orthodoxe et du trotskisme, cette parole critique s'élabore dans les termes du marxisme et selon le prisme ouvriériste. Au contact de l'expérience et de la parole prolétariennes, au fil des révolutions contre l'hydre totalitaire, de Berlin à Budapest, il faudra une dizaine d'années à Castoriadis et une partie du groupe $S$. ou $B$. pour parvenir à se déprendre peu à peu du carcan marxiste et s'apercevoir que l'approfondissement de leur diagnostic sur le régime russe faisait éclater ce cadre.

À travers la résistance au nazisme ${ }^{25}$ et au stalinisme, ce dont tous ces hommes firent l'expérience, c'est d'un langage fossilisé dans la certitude d'œuvrer dans le sens de l'histoire, au nom du pouvoir des peuples, langage accompagnant la liquidation féroce des opposants politiques ou des êtres jugés nuisibles et superflus. Leurs vies s'étaient trouvées passionnément engagées dans les combats pour une société débarrassée de l'oppression politique et de l'aliénation économique, elles se fracassaient devant la déformation monstrueuse de leurs idées dans l'imposture stalinienne. Ils rencontraient, dans les discours glaçants et la dialectique assassine $e^{26}$, les abîmes où s'effondraient les multiples chemins de l'autonomie.

J'ai cité ce que disait Orwell en 1946 concernant le langage politique moderne ; il faut aller plus loin. Derrière l'atteinte au langage, c'est la question de la possibilité même d'une vérité objective qui est en jeu. De retour d'Espagne, Orwell avait été saisi d'effroi, par la manière dont, de toutes parts, les journaux semblaient pratiquer une censure sélective. C'est là une des sources de sa

24 Vidal-Naquet, «Hommage", op. cit.

25 Impossible ici de faire une comparaison avec la résistance à la "langue nazie ", qui serait pourtant nécessaire. Je renvoie au livre indispensable de Jacques Dewitte, Le pouvoir de la langue et la liberté de l'esprit. Essai sur la résistance au langage totalitaire, Michalon, Paris, 2007.

26 Voir le poème de Paz "Pétrifiée pétrifiante " : "Les dialecticiens exaltent la subtilité de la corde / Les casuistes aspergent d'eau bénite les bourreaux / Nourrissent la violence de lait dogmatique... ", in Euvres, op. cit., p. 459. 
réflexion romanesque de 1984 sur l'appauvrissement systématique du langage comme visée de domination ultime et de disparition de toute pensée critique. Et au fond, résister aux mensonges publics, aux mystifications officielles et à l'impudeur journalistique, c'est tenter d'empêcher que soit « détruit le rapport des hommes à la signification, et le langage comme milieu et véhicule d'une vérité possible, donc d'un mouvement de la société ${ }^{27}$.

Castoriadis estimait que le langage est constitué de deux dimensions, entremêlées et irréductibles : le code et la langue, ou ce que j'appellerai pour ma part l'élément de code et l'élément poétique de toute langue. Le premier rend possible des relations stables, logiques et suffisamment définies entre les signes, entre les signifiants et les signifiés voire les référents ; nous sommes au plus près du code lorsque nous utilisons les mots de manière purement utilitaire et informative : "le train est à 8 heures ». Avec le second, s'ouvre la relation de signification, les renvois indéfinis à des " réalités " qui ne sont pas circonscrites dans des objets ou des phénomènes pleinement logiques et identifiables : «j’ai assis la Beauté sur mes genoux et je l'ai trouvée amère "... le sens vacille, explose en mille et une résonnances, s'ouvre multiplement sans qu'on puisse s'arrêter sur une seule détermination. Il y a du code dans toute poésie, et du poétique ou de la langue dans la parole la plus triviale, mais toute "parole parlante " (Merleau-Ponty) qui s'ouvre à la " polysémie féconde des mots ", qu'elle vienne de l'enfant, de l'amoureux, du poète, du philosophe ou des hommes en lutte pour leur liberté, est bien plus du côté du poétique que du code. Or que fait et que vise la langue totalitaire ou mieux la langue atteinte par la catastrophe totalitaire - en amont et en aval de son émergence ? Deux points retiennent l'attention de Castoriadis. Il y a d'abord la réduction des mots à des " marques", de purs « signaux d'activités-réflexes » : les signes « réactionnaire », « fasciste » ou " traitre " ne voulant rien dire d'autre que le comportement qu'ils déclenchent mécaniquement pour traiter les individus concernés en fonction du « code de traitement " qui leur est " applicable ". En second lieu, dans la mesure où la langue est vidée de sa substance et de toute signification véritable, « on peut faire avec les mots n'importe quoi, en changer arbitrairement et du jour au lendemain la relation avec "ce qu'ils désignent" ". Indépendamment des circonstances de l'analyse de Castoriadis au sujet du régime brejnévien, on voit bien ce qu'elle doit ici aux expériences et travaux antérieurs du résistant à la société bureaucratique et à la ruine du langage.

27 Castoriadis, Devant la guerre, Les réalités (1981), Le Livre de poche, "Biblio-essais ", Paris, 1983, p. 257-261 pour les passages cités. 


\section{De la marginalité à l'exploration de l'imagination. Éléments d'un problème}

La persévérance et la lucidité de l'aventure de Socialisme ou Barbarie, entourée aujourd'hui d'une aura mythique, ne doivent pas masquer la marginalité dans laquelle se trouvait le groupe, entouré par une véritable terreur intellectuelle exercée par le PCF et ses compagnons de route, accueilli par une " conspiration universelle du silence " (selon la formule de Castoriadis)... De même, Octavio Paz témoigne de la manière dont fut reçu le rapport qu’il avait composé et réussi à publier en 1951 à destination d'un public de langue espagnole au sujet des camps de concentration en Russie : son « intervention a été ignorée, ou comme on dit au Mexique, ninguneada, passée sous silence " - ce silence qui s'accompagne de la disqualification et d'une haine tenace... ${ }^{28}$

Dès 1946, les discussions enfiévrées entre Papaïoannou et Paz, arrivé également à Paris, concernent aussi la fameuse "question russe » et la "formule creuse " de Trotski sur l'URSS comme "État ouvrier dégénéré ». La liberté d'esprit du jeune $\mathrm{Grec}^{29}$, le milieu surréaliste parisien (où il retrouve Péret et rencontre André Breton) et, précisément, le procès intenté par David Rousset contre Les Lettres françaises à Paris en 1948 au sujet des témoignages sur les camps russes, finissent de dessiller les yeux d'Octavio Paz qui, poursuivant son exploration poétique et commençant son examen critique de la double identité mexicaine (espagnole et précolombienne), découvre en Inde (à la faveur d'un poste d'ambassadeur), une autre manière de mettre à distance les paroles mystificatrices du siècle, écho de la " force brute " (Castoriadis) et des illusions mortiferes. L'épreuve de l'incertitude et de la déchirure fut pour Octavio Paz l'école du " désapprendre " mais aussi celle de l'écriture : "Je m'embarquais dans chaque mot comme sur une coque de noix » ${ }^{30}$. Découvrant grâce à Victor Serge, dans la Partisan Review, les "Lettres de Londres » de George Orwell, Octavio Paz avait trouvé là une clarté, une sobriété et une audace de langage qui recoupaient ses propres élans poétiques : «Orwell s'était complètement débarrassé des maniérismes et byzantinismes dont souffraient mes amis, les marxistes ou les ex-marxistes français $»^{31}$. Il serait ici intéressant d'examiner de près la langue des militants de $S$. ou $B$. et de Castoriadis en particulier, dans les premières années du groupe, et de se demander dans quelle mesure elle rejoint cet effort de clarté et ce souffle nouveau auxquels aspirait Paz, et dans quelle mesure elle s'enfermait et les enfermait encore dans les carcans d'un certain byzantinisme.

28 Itinéraires, op. cit., p. 92.

29 Papaïoannou, L’idéologie froide. Essai sur le dépérissement du marxisme, J.-J. Pauvert, Paris, 1967 (dédié à Octavio Paz).

30 Itinéraires, op. cit., p. 92.

31 Ibid., p. 75. 
Le cours pris par les événements du monde et la manière dont $S$. ou B. en rend compte (révolutions prolétariennes à l'Est, mouvements de décolonisation, prémisses d'une contestation généralisée des autorités traditionnelles et du mode de vie en Occident, crise du régime en France...), conduisent, en 1958, à un rapprochement de Benjamin Péret avec les "socio-barbares "; et le poète surréaliste y retrouve avec plaisir un Chaulieu (pseudonyme de Castoriadis) " qui, entre-temps, a adopté à peu près toutes nos idées et s'est considérablement amélioré à tous les points de vue $»^{32}$. Malheureusement, la maladie fauche le fulgurant Péret et, si $S$. ou B. lui rend hommage en publiant Le déshonneur des poètes accompagné d'un article élogieux de Jean-Jacques Lebelen, 1959, il est frappant de constater le silence de Castoriadis et de ses commentateurs sur la source d'inspiration qu'ont pu représenter le surréalisme et les écrits de Benjamin Péret dans la vaste reconsidération de la place de l'imaginaire opérée par l'auteur de L'institution imaginaire de la société au tournant de 1960... Ni Breton, ni Péret ne sont cités pour " expliquer » cette évolution : ce sont Freud et l'ethnologie qui auraient été les plus déterminants répète-t-on depuis Castoriadis... Et pourtant, à relire Le déshonneur des poètes ou bien encore $\mathrm{La}$ parole est à Péret, force est de constater les intimes points de convergence : remise en cause de la dévalorisation multiséculaire de l'imagination comme puissance trompeuse, refus de l'opposition tranchée entre la logique et la raison d'un côté et l'intuition et la poésie de l'autre. On constate aussi le refus qui entraîne une réévaluation de leur source commune ('imaginaire), la reconnaissance de "l'exaltation collective réelle et profonde " à la source de toute parole authentiquement créatrice (ce que Castoriadis appellera "l'imaginaire instituant du collectif anonyme »), la dénonciation sans concession des barbaries et oppressions religieuses ou politiques qui «émasculent » la puissance poétique des êtres humains et les possibilités d'une « liberté entière et agissante " ${ }^{33} \ldots$

Castoriadis parle ici ou là des jeux surréalistes auxquels il se livrait à son arrivée à Paris et marque son admiration pour le surréalisme dans le texte des rencontres Octavio Paz en $1988^{34}$. Toutefois, ses écrits politiques et même philosophiques de l'après-guerre, marqués à la fois par l'ouvriérisme, le léninisme (du moins dans les premières années), le marxisme et la logique hégélienne, semblent assez peu témoigner de cette influence surréaliste, tout comme son refus, dans les années 60 , de dévier d'un sérieux militant et d'un style très

32 Benjamin Péret, Lettre du 17 juillet 1958 à Munis, in CEuvres complètes, t. 7, José Corti, Paris, 1995, p. 482.

33 Benjamin Péret, Le Déshonneur des poètes (Mexico, 1945) suivi de La parole est à Péret (New York, 1943), Mille et une nuits, Paris, 1996.

34 Rencontres en juin 1988 à Aix-en-Provence autour d'Octavio Paz (Détours d'écriture, Le temps des poètes, $n^{\circ}$ 13/14, "Octavio Paz ou la raison poétique », Aix-en-Provence, printemps/été 1989) ; l'intervention de Castoriadis et le dialogue qu'elle a suscité se trouve désormais dans "L'écrivain et la démocratie ", Fenêtre sur le chaos, op. cit. 
éloigné des " amusements » situationnistes. Il faudrait ici rapprocher cette sorte de "refoulement " de l'inspiration surréaliste avec les difficultés du groupe à changer ses manières de militer ${ }^{35}$. Alors que tout semble prêt pour « recommencer la révolution ", élargir le champ des préoccupations et des enquêtes à toutes les formes d'aliénation et de contestation de la société, au sein des universités, de la recherche, des rapports hommes-femmes, de la vie culturelle, des loisirs, etc., malgré des tentatives en ce sens, l'aventure s'épuise, la parole se tarit, comme le dira Daniel Blanchard, presque cinquante ans plus tard, dans Crise de mots ${ }^{36}$. L'auteur y dit l'ennui et l'étrangeté à soi qui remonte jusque dans la bouche et porte atteinte à la langue que l'on fait sienne, à la saveur des mots et au goût de la parole vive ; ou encore la montée d'une peur : celle du silence et de la parole vide, vidée par l'omniprésence d'une seule voix, celle de Castoriadis, «immense voix qui boit, qui boit ", reprend Blanchard d'Henri Michaux ; celle du vertige de cet état d'ennui et de cette montée du silence par contraste avec les années dans le " chaudron bouillonnant " de S. ou B. Un an après l'autodissolution du groupe, Mai 68 fait voler en éclat cet ennui ordinaire et fait surgir une " brèche ", immense « libération de la parole »...

\section{Du nihilisme et de l'insignifiance modernes. Interrogations}

Et pourtant, ce souffle inédit se fracasse aussi sur les impasses et les aveuglements politiques du maoïsme, la persistance des impostures staliniennes, puis, sur fond d'un singulier échec, sur " la montée " apparemment inexorable de "l'insignifiance " et du nihilisme marchand. C'est là le dernier moment de ces itinéraires croisés. Dès 1946, avant d'inventer le projet de la "novlangue " pour 1984 , Orwell ${ }^{37}$ avait clairement mis à jour et dénoncé la dégradation du langage dans les pays dits "démocratiques ", non pas au nom d'un archä̈que souci de correction, mais dans un but de clarté et de précision, à l'encontre de tous les confusionnismes et décervelages opérés à coup de slogans, de clichés et de mensonges. Reconnaître la lucidité dont Orwell, Paz ou Castoriadis ont fait preuve à l'égard du totalitarisme russe (et ses affiliés, en France comme en Amérique du Sud) comme des discours mystificateurs qui l'ont masqué ou justifié, cela signifie en même temps reconnaitre qu'ils ont tous décelé les racines du totalitarisme à l'œuvre dans la modernité, ne cessant de décrypter et dénoncer avec acrimonie les maux et les absurdités des sociétés occidentales.

35 Je renvoie le lecteur intéressé aux articles de Daniel Ferrand, Daniel Blanchard et Helen Arnold publiés dans les Cahiers Castoriadis n ${ }^{\circ}$ 7, Socialisme ou Barbarie aujourd'hui, Analyses et témoignages, S. Klimis, P. Caumières et L. Van Eynde (éd.), Facultés Universitaires Saint Louis, Bruxelles, 2012.

36 Éd. du Sandre, Paris, 2012. Fait remarquable, c'est notamment dans la poésie que Blanchard trouvera les ressources pour échapper à cet épuisement...

37 "La politique et la langue anglaise ", op. cit. 
Reprenant le mot de Péret, Paz estimait que la parole au XX $X^{\mathrm{e}}$ siècle avait été multiplement "déshonorée ": les mots ordinaires pas moins que les mots de l'émancipation et de la politique (volonté, égalité, peuple, souveraineté, socialisme, révolution...), que ce soit par les monstruosités totalitaires ou que ce soit par «l'écœurante cucuterie de la publicité - toute cette rhétorique asphyxiante à la fois nauséabonde et sucrée des gens satisfaits et engourdis par la trop bonne chère $»^{38}$. Dans le dernier chapitre de son autobiographie intellectuelle, dans des pages écrites au moment où s'écroule de l'intérieur ce monstre totalitaire russe que le monde libéral avait cherché à contenir, voulant le croire issu d'une source toute extérieure à lui-même, le poète mexicain reprend sommairement cette critique du nihilisme marchand. Même si Paz s'est alors rallié au libéralisme politique, il ne continue pas moins à s'inquiéter de ce que porte le nihilisme : " conformisme universel ", nivellement sans saveur opéré par la seule valeur comptable, anesthésie et somnolence engendrées par l’omniprésence de la publicité, la vie des hommes est comme " dépeuplée ", " la véritable vie court à vau-l'eau » : "Nos aïeules récitaient d'interminables rosaires; nos filles répètent des slogans publicitaires ${ }^{39}$. Tel est bien le péril qui guette la parole moderne : que les hommes ne soient plus que les ventriloques d'eux-mêmes, pas même désireux de répondre de leurs propres mots...

Tout au long des trente dernières années de sa vie, bien qu'éloigné du militantisme, l'auteur de La montée de l'insignifiance maintient de son côté un projet de "transformation radicale de la société " et n'a de cesse de ferrailler contre les « divertisseurs " et toutes les manifestations d'une dégradation de la parole dans l'espace public. Ainsi, dans les années 70, lorsque les «nouveaux philosophes " travestissent et émasculent les critiques du marxisme et du totalitarisme tout en étant l'objet d'un incroyable engouement médiatique, Castoriadis ne se contente pas de dénoncer ce dont ils font leur commerce, il y voit les symptômes d'une société rongée par " les lobbys et les hobbies » et se demande douloureusement : " sous quelles conditions sociologiques et anthropologiques, dans un pays de vieille et grande culture, un "auteur" peut-il se permettre d'écrire n'importe quoi, la "critique" le porter aux nues, le public le suivre docilement - et ceux qui dévoilent l'imposture, sans nullement être réduits au silence ou emprisonnés, n'avoir aucun écho effectif ? $"{ }^{40}$. Cette interrogation traduit l'incrédulité et la volonté de résister à cette véritable corruption de l'espace de débat par l'industrie publicitaire : "Plus insidieuse, l'imposture publicitaire n'est pas, à la longue, moins dangereuse que l'imposture totalitaire. Par des moyens différents, l'une et l'autre détruisent

38 "Post-scriptum ", cité dans Solo à deux voix, op. cit., p. 27.

39 Itinéraires, op. cit., p. 144 et 145.

40 "L'industrie du vide" (1978), in Domaines de l'homme. Les Carrefours du labyrinthe II, Seuil, Paris, 1986, p. 32-40. 
l'existence d'un espace public de pensée, de confrontation, de critique réciproque $»^{41}$. Au-delà du dérisoire, se manifestent pour Castoriadis plusieurs traits des sociétés contemporaines qui demandent à être réfléchis et combattus : d'abord, dans la continuité de cinquante années d'imposture totalitaire, une profonde usure des mots et une "corruption des mécanismes mentaux " (de critique, de discernement, de responsabilité de la parole, de pudeur aussi dans l'énoncé des jugements, etc.), ensuite l'envahissement du terrain des idées et du débat intellectuel par la logique marchande qui émousse et récupère toute critique, tout en assurant la promotion du pire ou de l'insignifiant ${ }^{42}$. Mais cela ne peut perdurer et s'approfondir que par l'adhésion ou l'indifférence des individus ainsi que leur repli dans la vie privée, laissant libre champ à la " contre-offensive " libérale et oligarchique. Un dernier paradoxe retient l'attention. Si Paz rejoint à la fin de sa vie une sorte de "tocquevilisme " critique qui tempère son constat sur le nihilisme, Castoriadis pour sa part fait preuve d'un pessimisme toujours plus radical : sans renier ses aspirations révolutionnaires, il ne voit plus quapathie et privatisation du côté des "zappeurs abrutis» des sociétés occidentales, réunis en un même bloc. En dépit des nuances qu'il apporte ici ou là à son diagnostic, tout se passe comme si, à partir des années 80 , Castoriadis s'était coupé de la complexité du réel et de la vie ordinaire. Comme oublieux de l'inspiration féconde de $S$. ou B., il ne semble plus voir ni vouloir explorer la manière dont les hommes, dans leur diversité, peuvent résister, y compris sur le terrain des mots, à leur transformation en agents passifs du mouvement en avant de la technoscience et du monde marchand. Et pourtant, Castoriadis savait, avec Paz, que le rôle de l'écrivain, poète ou philosophe, n'était pas seulement de parler mais d' " écouter " et de redonner au monde toute "sa présence " ${ }^{43} \ldots$

Le courage et la lucidité dont firent preuve Octavio Paz et Cornelius Castoriadis pour résister à la corruption du langage ont ceci d'exemplaire qu'ils ne nous laissent pas démunis pour demeurer vigilants à l'égard de nos propres habitudes linguistiques. Du rire à l'appel au sens commun, de l'ironie à la colère, de ce que Paz appelait «l'hygiène mentale » aux ressources de la critique et de la poésie, le poète mexicain et le philosophe venu d'Athènes nous auront placés dans une dette incommensurable devant la monstruosité totalitaire et les ravages publicitaires. Mais cette exemplarité n'a rien d'un modèle et requiert la critique. Ainsi, lorsqu’à partir de 1994 une voix singulière s'est levée au Chiapas, celle des insurgés zapatistes, Octavio Paz n’a voulu y voir qu'une énième manifestation des guérillas sanglantes du siècle sur le continent sud-américain ; et de son côté, Castoriadis s'est d'abord méfié de ce qu'il croyait

41 Ibid.

42 La montée de l'insignifiance, Seuil, Paris, 1996.

43 Voir, à nouveau, le dialogue avec Octavio Paz, in "L'écrivain et la démocratie », op. cit., p. 97. 
être un " coup " médiatique. Et pourtant, des montagnes mexicaines montait un chant nouveau de résistance et d'autonomie : " notre cœur est joyeux car nous parlons en silence, nous nous montrons en nous cachant..."

Laurent Leylavergne

EA $4574 \mathrm{SPH}$

Université Bordeaux Montaigne

Laurent.Leylavergne@ac-poitiers.fr

\begin{abstract}
Résumé
Mon propos est consacré à la question de la résistance à la corruption du langage au XXe siècle à travers les œuvres et les parcours du poète Octavio Paz et du philosophe Cornelius Castoriadis, liés par une estime réciproque et des préoccupations communes, dissidents inclassables contre les langues totalitaires ou la marchandisation des mots et des idées. Cela me conduit à une réflexion conjointe sur la nature du langage et la création poétique.
\end{abstract}

\title{
Mots-clés
}

Langage, imagination, poésie, politique, totalitarisme.

\begin{abstract}
My aim relates to the question of resistance to the corruption of language during the $20^{\text {th }}$ century through works and courses of the poet Octavio Paz and the philosopher Cornelius Castoriadis, bound by a reciprocal regard and common concerns, unclassifiable dissidents against the totalitarian languages or the commodification of words and ideas. That leads me to a joint thought on the nature of language and poetic creation.
\end{abstract}

\section{Keywords}

Language, imagination, poetry, politics, totalitarianism. 